\title{
Epidémiologie de Diarrhée des Veaux dans les Troupeaux Bovins au Sud du Togo
}

\section{Kotoe Mensah Délako, (DVM/MS) (Auteur Correspondant)}

Institut Togolais de Recherche Agronomique; Centre de Recherche Agronomique Zone Forestière, Station Avétonou, Kpalime, Togo

Seme Kpassi, (PhD, Dr., Ing.)

Institut Togolais de Recherche Agronomique ; Centre de Recherche

Agronomique Zone Forestière, Station Avétonou, Kpalime, Togo

Ecole Supérieure d'Agronomie, Département des Sciences Animales et

Vétérinaires, Université de Lomé, ESA/UL Lomé, Togo

Kossoga Kakom, (Ing/MS)

Institut Togolais de Recherche Agronomique ; Centre de Recherche Agronomique Zone Forestière, Station Avétonou, Kpalime, Togo

\section{Teteh Ayivi, (DMV/PhD)}

Ecole Supérieure d'Agronomie, Département des sciences Aviaires, Université de Lomé; Lomé, Togo

Pato Pidemnéwé, (DMV/MS)

Direction de l'élevage ; Ministère de l'Agriculture, de l'Elevage, et des Ressources Halieutiques Lomé, Togo

Awuni Joseph, (DVM/MS)

Laboratoire Vétérinaire d'Accra; Veterinary Services, Accra, Ghana

Doi:10.19044/esj.2020.v16n27p213 URL:http://dx.doi.org/10.19044/esj.2020.v16n27p313

\section{Résumé}

On observe depuis quelques temps dans les troupeaux bovins au sud du Togo, l'émergence de certaines pathologies dont la plus préoccupante est la diarrhée des veaux (D.V). Cette diarrhée provoque une forte mortalité surtout chez les jeunes âgés de 0 à 6 mois. Ceci a pour corollaire un faible taux de croissance des effectifs des troupeaux. Dans cette optique, le but de l'étude a été de faire l'état des lieux afin de déterminer les causes de la diarrhée. Pour cela, des enquêtes épidémiologiques, suivies d'analyses bactériologiques et parasitologiques ont été conduites dans les élevages de 3 préfectures : Agou, Haho et Ave, et ont porté sur un échantillon de 46 troupeaux avec un effectif de 3381 têtes de bovin et 1035 veaux. Les indicateurs épidémiologiques ont révélé une prévalence moyenne de diarrhée des veaux de $40 \%$ dans la zone d'étude et le taux de mortalité global (toute mortalité comprise) des veaux a 
été de $30 \%$. Parmi les veaux morts, $77,12 \%$ a été causée par la DV. Plus de 60 $\%$ des troupeaux visités ont connu un taux de mortalité des veaux supérieur à $20 \%$. Les causes directes de la diarrhée ont été multifactorielles et concernent entre autres les entérobactéries (Escherichia Coli, Salmonella sp.) et les parasites gastro-intestinaux (Strongles, Eimeria spp.). Les mauvaises pratiques d'hygiène et une absence de suivi zoo-sanitaire ont été également les causes majeures de cette épidémiologie.

Mots-clés : Diarrhée, Mortalité, Enquête, Infection, Troupeaux

\section{Epidemiology of Calf Diarrhea in Cattle Herds in Southern Togo}

Kotoe Mensah Délako, (DVM/MS) (Auteur Correspondant)

Institut Togolais de Recherche Agronomique; Centre de Recherche

Agronomique Zone Forestière, Station Avétonou, Kpalime, Togo

\section{Seme Kpassi, (PhD, Dr., Ing.)}

Institut Togolais de Recherche Agronomique ; Centre de Recherche

Agronomique Zone Forestière, Station Avétonou, Kpalime, Togo

Ecole Supérieure d'Agronomie, Département des Sciences Animales et

Vétérinaires, Université de Lomé, ESA/UL Lomé, Togo

Kossoga Kakom, (Ing/MS)

Institut Togolais de Recherche Agronomique ; Centre de Recherche

Agronomique Zone Forestière, Station Avétonou, Kpalime, Togo

\section{Teteh Ayivi, (DMV/PhD)}

Ecole Supérieure d'Agronomie, Département des sciences Aviaires,

Université de Lomé; Lomé, Togo

Pato Pidemnéwé, (DMV/MS)

Direction de l'élevage ; Ministère de l'Agriculture, de l'Elevage, et des

Ressources Halieutiques Lomé, Togo

Awuni Joseph, (DVM/MS)

Laboratoire Vétérinaire d'Accra; Veterinary Services, Accra, Ghana

\section{Abstract}

Some time ago, observations made in bovine herds in the southern part of Togo revealed an outbreak of new pathologies among which the calf diarrhea (CD) was the most important. This diarrhea caused a high mortality among the young calves, particularly those within 0 to 6 months of age. As a 
result, there is a low rate of increment in the number of herds. This paper focuses on highlighting the point and finding the causes of the disease. An epidemiological survey followed by bacteriological and parasitological analysis was carried out on a sample of 46 herds with 3381 animals with 1035 calves in the prefecture of Agou, Haho, and Ave for assessment. The epidemiological indicators revealed an average prevalence of $40 \%$ in the area of the study, and the global mortality rate was $22 \%$ among which $77.12 \%$ of the mortality was caused by CD. More than $60 \%$ of the herds visited recorded a mortality rate higher than $20 \%$. The major causes of the CD have been multifactorial, which constitutes enterobacterial infections (E. Coli, Salonellae sp.) and gastro intestinal worms (Strongles, Emeiria spp.). Poor hygiene practices and a lack of zoo-sanitary monitoring were also the major causes of this epidemiology.

Keywords: Diarrhea, Mortality, Investigation, Infection, Herds

\section{Introduction}

Le cheptel bovin traditionnel ne peut acquérir son plein épanouissement que dans un environnement saint et confortable où les problèmes sanitaires et alimentaires sont absents. Ces deux facteurs sont déterminants en élevage traditionnel mais sont fortement dépendants des conditions climatiques. La maitrise des facteurs climatiques pour une bonne alimentation d'une part, et des facteurs sanitaires d'autres parts, participent au bon développement du troupeau, améliorent la production de lait et donnent une bonne croissance aux veaux et par conséquence contribuent à l'accroissement de la taille du troupeau. Ces derniers, au demeurant très sensibles à ces problèmes sanitaires, constituent dans une large mesure le noyau sur lequel toute projection statistique se base pour l'agrandissement du troupeau.

Cependant, depuis quelques années une diarrhée profuse et sanguinolente se terminant souvent par la mort a été déclarée dans les troupeaux dans la Région des Plateaux au Togo. Les veaux âgés de 0 à 6 mois ont été les plus sensibles et environ la moitié de ces veaux atteignent rarement 12 mois d'âge (Anderson et al., 2001). Bien que des efforts de suivi zoosanitaire aient été faits, les résultats escomptés n'ont pas été satisfaisants et les causes fondamentales de la maladie n'ont pas été élucidées. La mortalité des veaux devient alors l'une des causes principales du faible taux de croissance observé dans les troupeaux dans toute la région créant ainsi, d'une part un manque à gagner en viande et en lait, et d'autre part une baisse du revenu du paysan. 
Certes la diarrhée ne constitue pas en elle-même une maladie spécifique (Bradford \& Smith, 2008 ; Hunter et al., 1994) mais a des causes multiples (Mae, 2010 ; Dufrasne, 2003 ; Morin et al., 1976). Le diagnostic devient alors très complexe et nécessite une approche intégrée de méthodes. L'objectif de cette étude est alors de faire l'état des lieux et de déterminer les origines de la diarrhée. Plus spécifiquement il s'agit de déterminer les causes principales à travers les recherches bactériologiques, parasitologiques, les indicateurs épidémiologiques et les causes favorisantes par des enquêtes dans trois préfectures au sud du Togo.

\section{Matériel et méthodes}

Cette étude a été conduite d'abord par la recherche des causes favorisantes suivie des causes principales de la DV. Une analyse inter- action de ces deux paramètres a permis de faire une interprétation assez fiable des causes de la DV.

\section{Zone d'étude}

L'étude a été menée au sud du Togo dans les préfectures d'Agou, d'Avé et de Haho localisées dans les Régions des Plateaux et Maritime. Il s'agit d'une zone montagneuse dominée par les monts Agou culminant à $986 \mathrm{~m}$. La zone jouit d'un climat de type soudano-guinéen avec une grande saison sèche qui s'étend de novembre à février et une petite en juillet - août ; deux saisons pluvieuses qui s'étendent de mars à juin et en septembre -octobre. La pluviométrie moyenne annuelle varie entre 1100 et $1400 \mathrm{~mm}$ avec les plus grandes précipitations en juin. La végétation est constituée par un ensemble de savanes arborées traversées par un endroit de forêts galeries. La faune sauvage est constituée d'antilopes, de biches, de phacochères, de rongeurs et de reptiles.

\section{Les causes favorisantes de la diarrhée}

Cette étude a été faite par des enquêtes et par des appréciations du mode d'élevage dans les troupeaux des préfectures d'Agou, de l'Avé, de Haho. Les enquêtes se sont déroulées du $1^{\text {er }}$ mars au 30 avril (faible saison de vêlage) puis du 15 octobre au 30 décembre (période de vêlage intense) 2016 dans 45 troupeaux de bovins traditionnels et un (1) troupeau dans une station d'élevage à Avétonou située dans la préfecture d'Agou au Centre de Recherche Agronomique zone Forestière (CRAF) de l'Institut Togolais de Recherche Agronomique (ITRA).

$\mathrm{Au}$ cours de l'enquête, des interviews ont été menées auprès des éleveurs à travers des questionnaires qui ont été développés afin d'obtenir des informations sur le mode d'élevage et l'effectif du troupeau, l'effectif des veaux avec leur âge et l'alimentation des veaux. Les critères de choix d'un 
troupeau ont été la taille de ce dernier (en moyenne de 25 à 100 têtes), les vêlages ayant lieu entre le $1^{\mathrm{er}}$ janvier et le 30 décembre 2016 , et la présence de cas de diarrhée et de mortalité des veaux due ou non à la $\mathrm{D}$. $\mathrm{V}$ durant la période de l'étude. Au total 46 troupeaux ont été retenus pour la conduite de l'étude. Ils étaient de race taurine et de zébu composé de 3381 têtes dont 691 d'Agou, 938 d'Avé, 1727 de Haho, et 25 d'Avétonou. Au sein de ces troupeaux, 1035 veaux dont 237 d'Agou, 118 d'Avé, 588 de Haho, et 22 d'Avétonou ont été recensés. Parmi les veaux, vingt (20) souffrant cliniquement de diarrhée ont été choisis de façon aléatoire dans différents troupeaux puis identifiés par des numéros fixés à l'oreille pour des prélèvements de selles et de sang pour des analyses bactériologiques et parasitologiques. Ils ont été repartis suivant les classes d'âge : 0 à 1 mois; 1 à 3 mois; 3 à 6 mois ; 6 mois et plus, conformément aux regroupements effectués par les bouviers dans les étables traditionnelles du milieu.

Les cas de diarrhée clinique dans les troupeaux ont été notifiés par la présence de fécales liquides ou pâteux sur le sol ou au niveau de l'anus des veaux avec présence de souillure d'excréments liquides.

Le mode d'élevage dans les troupeaux a été apprécié en considérant les pratiques d'alimentation, de conduite, du suivi sanitaire, et de consommation du colostrum par le veau dans les différents troupeaux. Le mode d'élevage a été également étudié à travers la mise en place de deux groupes de troupeau (troupeaux aux pratiques d'élevage identiques et troupeaux aux pratiques d'élevage divergentes) et leur comparaison.

En fin, le mode d'élevage a été évalué et apprécié à travers les taux de mortalité obtenus dans chaque troupeau. Pour ce faire, des troupeaux ont été identifiés et désignés par la lettre $\mathrm{A}, \mathrm{B}$, ou $\mathrm{C}$ représentant les catégories de troupeaux et respectant les critères suivants :

Troupeaux de catégorie $\mathrm{A}$; troupeau à mortalité très forte: (Taux de mortalité : $\mathrm{Td}>20 \%$ );

Troupeaux de catégorie $\mathrm{B}$; troupeau à mortalité moyenne: (Taux de mortalité : $10 \%<\mathrm{Td}<20 \%$ );

Troupeaux de catégorie $\mathrm{C}$; troupeau à mortalité faible : (Taux de mortalité : $\operatorname{Td}<10 \%$ ).

Ils sont par ailleurs, considérés comme des troupeaux de référence auxquels les autres troupeaux ont été comparés.

\section{Les causes principales de la diarrhée des veaux}

Cette étude a été effectuée par la détection des charges bactériologiques et parasitologiques au laboratoire. Pour ce faire, l'étude s'est basée sur des prélèvements de selles et de sang sur 20 veaux choisis au hasard dans les différents troupeaux et présentant des signes cliniques de DV. 
Les prélèvements de selles pour la coprologie ont été exécutés par enfoncement du doigt après habillage de gant directement dans l'anus des veaux. Les selles recueillies sont emballées dans des sachets plastiques et convoyées sous frais au laboratoire.

Quant au sang, il a été recueilli dans des tubes Vacutainers héparinés après désinfection et ponction de la veine jugulaire. Chaque prélèvement a été identifié par des étiquettes portant le numéro du veau.

\section{Examens parasitologiques}

La technique d'analyse coprologique a été exécutée par observation macroscopique et microscopique. L'observation microscopique a été faite à l'aide d'un microscope de marque Zeiss et de grossissement $10 \times 10$. La méthode de flottaison dans une solution de Nacl à $40 \%$ suivant la technique décrite par Willis (1983) et Thiempont et al. (1995) a été utilisée. La cellule de Mc Master a servi pour le comptage des œufs des parasites (OPG). L'observation macroscopique a porté sur l'état physique, la consistance et la couleur des selles.

\section{Examens bactériologiques}

La technique microbiologique a porté à la fois sur les fèces et sur le sang. Les méthodes bactériologiques classiques décrites par Widal et Félix (2009) ont été utilisées pour la recherche des entérobactéries dans le sang. Quant aux selles, les milieux de culture de bouillon sélénites de gélose S.S et de E.M.B ont été utilisés pour la culture et l'isolément des bactéries (Mae, 2010). Les micro-organismes recherchés ont été les germes entéro-pathogènes de Escherichia coli et de Salmonelle sp. Les titres d'anticorps $<\mathrm{T}>$ de ces germes ont été évalués et ont permis d'apprécier la charge bactérienne des microbes. Pour cette étude, seuls les germes dont les titres d'anticorps T $>1: 200$ ont été retenus et considérés comme véritablement pathogènes (Bradford \& Smith, 2008).

\section{Indicateurs épidémiologiques \\ Prévalences de la DV}

Prévalence globale de la DV dans le troupeau.

Elle a pu être étudiée après le recensement de l'effectif des veaux nés et celui des veaux malades dans le troupeau.

C'est le rapport entre l'effectif des veaux nés et les veaux malades (toutes maladies comprises) dans le troupeau en pourcentage.

Nombre de veaux malades

Prévalence globale $(\mathrm{Pg})=$ $X 100(1)$

Effectif total des veaux nés dans le troupeau au cours de la période 
Prévalence de la DV

Elle a été évaluée suite au recensement des veaux malades de diarrhée ou ayant présenté des symptômes de diarrhées.

C'est le rapport entre le nombre de cas de diarrhée clinique de veau et l'effectif total des veaux nés dans le troupeau au cours de la période de l'étude en pourcentage.

Prévalence diarrhée $(P d v)=$

Nombre de cas de DV

Effectif total des veaux nés dans le troupeau au cours de la période

\section{Taux de mortalité}

Taux de mortalité global

Il a été évalué après recensement de l'effectif du troupeau et celui des veaux morts

C'est le rapport entre l'effectif des veaux morts (toute mortalité confondue) et l'effectif des veaux nés dans le troupeau au cours de la période en pourcentage.

Taux de mortalité globale $(\mathrm{Tg})=$

Nombre de veaux morts

Effectif total des veaux nés dans le troupeau au cours de la période $X 100(3)$

Taux de mortalité dû à la D.V

Cet indicateur a été évalué en considérant les veaux morts avec des symptômes de diarrhées.

C'est le rapport entre le nombre de veaux morts de DV et l'effectif des veaux nés dans le troupeau au cours de la période en pourcentage.

Nombre de veaux morts de $D V$

Taux de mortalité dî à la $\mathrm{DV}(\mathrm{Td} v)=$ $X 100(4)$

Effectif total des veaux nés dans le troupeau au cours de la période

Part des veaux morts de D.V par rapport à l'ensemble des veaux morts (toute pathologie comprise) dans le troupeau.

Ce taux a été obtenu suite au rapport entre les veaux morts de diarrhée et l'ensemble des veaux morts dans le troupeau. Il indique par ailleurs le taux de menace de la DV dans le troupeau.

Taux des veaux morts de diarrhée (Tdv)

Taux de menace de la diarrhée $(T s)=$ $X 100(5)$

Taux des veaux morts dans le troupeau (Tm)

\section{Résultats}

Facteurs favorisants

\section{Recensement des troupeaux et des veaux}

Les veaux représentent en moyenne 31\% de l'effectif du troupeau (Tableau 1) 
Tableau 1. Recensement et représentation des veaux dans les troupeaux.

\begin{tabular}{|l|l|l|l|l|}
\hline $\begin{array}{c}\text { Sites de } \\
\text { l'expérimentation }\end{array}$ & $\begin{array}{c}\text { Nombre de } \\
\text { troupeaux }\end{array}$ & $\begin{array}{c}\text { Effectif } \\
\text { d'animaux } \\
\text { dans les } \\
\text { troupeaux }\end{array}$ & $\begin{array}{c}\text { Effectif de } \\
\text { veaux dans } \\
\text { les } \\
\text { troupeaux }\end{array}$ & $\begin{array}{c}\text { Pourcentage } \\
\text { veaux (\%) }\end{array}$ \\
\hline Agou & 10 & 691 & 237 & 34 \\
\hline Ave & 15 & 938 & 188 & 20 \\
\hline Haho & 20 & 1727 & 588 & 34 \\
\hline Avétonou & 1 & 25 & 22 & 88 \\
\hline Total & 46 & 3381 & 1035 & 31 \\
\hline
\end{tabular}

Tableau 2. Recensement et répartition des veaux morts suivant les tranches d'â

\begin{tabular}{|l|c|c|c|c|c|c|c|}
\hline $\begin{array}{c}\text { Site de } \\
\text { l'expérime } \\
\text { ntation }\end{array}$ & $\begin{array}{c}\text { Effectif } \\
\text { Veaux(n) }\end{array}$ & $\begin{array}{c}\text { Effectif } \\
\text { veaux } \\
\text { malades } \\
\text { de DV }\end{array}$ & $\begin{array}{c}\text { Effectif } \\
\text { veaux } \\
\text { morts de } \\
\text { DV }\end{array}$ & \multicolumn{5}{|c|}{\begin{tabular}{l}
\multicolumn{5}{|c|}{ Effectif veaux morts de DV selon les tranches } \\
d'âge (mois)
\end{tabular}} \\
\cline { 2 - 8 } & & & & $0-1$ & $1-3$ & $3-6$ & $>6$ \\
\hline Agou & 237 & 96 & 72 & 36 & 23 & 12 & 1 \\
Avé & 188 & 47 & 30 & 17 & 7 & 3 & 3 \\
Haho & 588 & 177 & 129 & 61 & 45 & 15 & 08 \\
Avétonou & 022 & 14 & 5 & 3 & 2 & 00 & 00 \\
Région & 1035 & 414 & 236 & 125 & 78 & 26 & 7 \\
\hline
\end{tabular}

Répartition des troupeaux par catégorie et suivant le mode d'élevage

La catégorie des troupeaux est fonction du mode d'élevage et du taux de mortalité. (Tableau 3)

Tableau 3. Comparaison des catégories de troupeaux selon le mode d'élevage

\begin{tabular}{|c|c|c|c|c|}
\hline \multirow[t]{2}{*}{ Rubriques } & \multirow[t]{2}{*}{ Modes d'élevage } & \multicolumn{3}{|c|}{ Catégories de troupeaux } \\
\hline & & $\mathbf{A}$ & $\mathbf{B}$ & $\mathbf{C}$ \\
\hline \multirow{4}{*}{$\begin{array}{l}\text { Pratiques } \\
\text { identiques }\end{array}$} & Pâturage naturel & oui & Oui & Oui \\
\hline & Parc de nuit & oui & Oui & Oui \\
\hline & Traite du lait & oui & Oui & Oui \\
\hline & Fourrage naturel & oui & Oui & Oui \\
\hline \multirow{9}{*}{$\begin{array}{l}\text { Pratiques } \\
\text { divergentes }\end{array}$} & $\begin{array}{l}\text { Compléments } \\
\text { alimentaires }\end{array}$ & Non & Oui & oui \\
\hline & Eau de boisson & Marre, barrage & Marre, barrage & Puits, eau de pluie \\
\hline & Habitat & Inexistant & Matériaux de fortune & $\begin{array}{l}\text { Bâtiment en tôle } \\
\text { ou en paille }\end{array}$ \\
\hline & Plancher/étable & Bouse de vache & Parfois damé & Béton ou ciment \\
\hline & Conduite veaux & Avec leur mère & $\begin{array}{l}\text { Parc de nuit et } \\
\text { pâturage }\end{array}$ & Au parc pour veau \\
\hline & Hygiène du parc & Pas de nettoyage & Une fois par trimestre & $\begin{array}{l}\text { Une fois par } \\
\text { semaine }\end{array}$ \\
\hline & Suivi sanitaire & $\begin{array}{lll}\begin{array}{l}\text { Ecorce } \\
\text { d'arbres }\end{array} & \text { racine } \\
\end{array}$ & $\begin{array}{l}\text { Rarement ou gélules de } \\
\text { tétracycline }\end{array}$ & $\begin{array}{l}2 \text { fois par trimestre } \\
\text { par un technicien }\end{array}$ \\
\hline & Traite du lait & Obligatoire & Pas régulière & Inexistante \\
\hline & Prise du colostrum & Pas de surveillance & - & Assistance au veau \\
\hline
\end{tabular}




\begin{tabular}{|l|l|l|l|l|}
\hline & $\begin{array}{l}\text { Caractéristiques du } \\
\text { troupeau }\end{array}$ & $\begin{array}{l}\text { Propriétaires } \\
\text { multiples }\end{array}$ & Individuel & Individuel \\
\cline { 2 - 4 } & $\begin{array}{l}\text { Catégories/mode } \\
\text { d'élevage (\%) }\end{array}$ & 60 & 18 & 22 \\
\hline $\begin{array}{l}\text { Taux de mortalité des } \\
\text { Veaux malades de } \\
\text { de DV : Td (\%) }\end{array}$ & $\mathrm{Td}>20 \%$ & $10 \%<\mathrm{Td}<20 \%$ & $\mathrm{Td}<10 \%$ \\
\hline
\end{tabular}

\section{Indicateurs épidémiologiques}

\section{Prévalence de la maladie}

La prévalence de la DV dans les deux régions a fluctué entre 25 et $64 \%$ avec une moyenne de $40 \%$. La station d'Avétonou a enregistré la plus forte prévalence (64\%) (Figure 1).

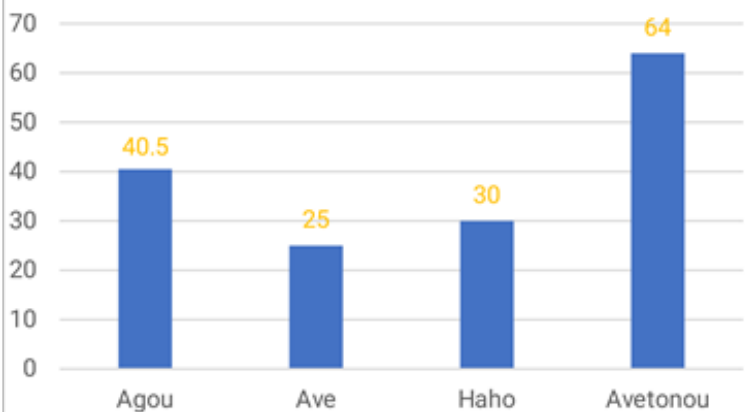

Figure 1. Prévalence de la maladie dans les différentes préfectures et à Avétonou

\section{Taux de mortalité global et taux de mortalité dû à la diarrhée}

Parmi les veaux nés, $30 \%$ meurent avant l'âge de 12 mois et $77,12 \%$ des mortalités est dû à la DV (Tableau 4).

Tableau 4. Taux de mortalité global et taux de mortalité due à la diarrhée des veaux (\%)

\begin{tabular}{|c|c|c|c|c|c|c|}
\hline Site & $\begin{array}{c}\text { Effectif } \\
\text { veaux nés } \\
(\mathbf{n})\end{array}$ & $\begin{array}{c}\text { Effectif } \\
\text { veaux morts } \\
(\mathbf{E m})\end{array}$ & $\begin{array}{c}\text { Effectif } \\
\text { Veaux morts } \\
\text { de diarrhée } \\
\text { (Edv) }\end{array}$ & $\begin{array}{c}\text { Taux de } \\
\text { mortalité } \\
\text { global (Tg) } \\
\mathbf{\%}\end{array}$ & $\begin{array}{c}\text { Taux des } \\
\text { veaux } \\
\text { mortalité de } \\
\text { diarrhée } \\
\text { (Tdv)\%) }\end{array}$ & $\begin{array}{c}\text { Nombre de veaux } \\
\text { morts de diarrhée } \\
\text { sur nombre total } \\
\text { de veaux morts } \\
\text { Ts=Edv/Em (\%) }\end{array}$ \\
\hline Agou & 237 & 89 & 72 & 38 & 30 & 81 \\
\hline Ave & 188 & 41 & 30 & 22 & 16 & 73 \\
\hline Haho & 588 & 171 & 129 & 29 & 23 & 75 \\
\hline Avétonou & 22 & 5 & 5 & 23 & 23 & 100 \\
\hline Région & 1035 & 306 & 236 & 30 & 23 & 77,12 \\
\hline
\end{tabular}

$\mathrm{Tg}=$ Taux de mortalité globale ; Edv= Effectif veaux morts de diarrhée ; Tm= effectif veaux morts.

\section{Taux de mortalité suivant l'âge des veaux}

Dans les deux régions prospectées, $86 \%$ des veaux atteints de la diarrhée meurent entre 0 et 3 mois d'âge, mais la majorité (53\%) succombe 
entre 0 et 1 mois d'âge. La mortalité des veaux due à la diarrhée va decrescendo avec l'âge ; elle est de $53 \%$ avant 1 mois et de $3 \%$ à 6 mois et plus. La sensibilité des veaux à la diarrhée diminue avec l'âge dans la région (Tableau 5).

\begin{tabular}{|l|l|c|l|l|l|l|}
\hline Tableau 5. Taux de & \multirow{2}{*}{$\begin{array}{c}\text { Effectif } \\
\text { mortalité des veaux } \\
\text { morts de DV suivant } \\
\text { les classes d'âge } \\
\text { (\%)Site de }\end{array}$} & $\begin{array}{c}\text { Effectif } \\
\text { nés }\end{array}$ & $\begin{array}{c}\text { ceaux morts } \\
\text { de diarrhée }\end{array}$ & & & \multicolumn{4}{|c|}{ Classe d'âge (mois) } \\
\cline { 5 - 7 } & & & $1-3$ & $3-6$ & $>6$ \\
\hline L'expérience & & & & & & \\
Agou & 237 & 72 & 50 & 32 & 17 & 1 \\
Ave & 188 & 30 & 56 & 23 & 11 & 10 \\
Haho & 588 & 129 & 47 & 35 & 12 & 06 \\
Avétonou & 022 & 5 & 60 & 40 & 00 & 00 \\
Région & 1035 & 236 & 53 & 33 & 11 & 03 \\
\hline
\end{tabular}

Répartition des troupeaux traditionnels par catégorie et en fonction des taux de mortalité due à la DV

Plus de la moitié des troupeaux (en moyenne 60\%) est de la catégorie A, avec un taux de mortalité $\mathrm{Td}>20 \%$. Seul $22 \%$ des troupeaux appartiennent à la catégorie $\mathrm{C}$ avec un taux de mortalité $\mathrm{Td}<10 \%$.

La catégorie B représente $18 \%$ de l'élevage avec un taux de mortalité situé entre $10 \%<\mathrm{Td}<20 \%$ (Tableau 6).

Tableau 6. Catégorie de troupeaux selon le taux de mortalité dû à la diarrhée

\begin{tabular}{|c|c|c|c|c|c|c|c|}
\hline & & \multicolumn{6}{|c|}{ Catégories de troupeaux } \\
\hline \multirow{3}{*}{ Site } & \multirow{3}{*}{\begin{tabular}{c} 
Nombre de \\
troupeaux \\
\cline { 3 - 8 }
\end{tabular}} & \multicolumn{2}{|c|}{$\mathrm{A}$} & \multicolumn{2}{|c|}{$\mathrm{B}$} & \multicolumn{2}{c|}{$\mathrm{C}$} \\
\cline { 3 - 8 } & recensés & $\mathrm{T}>20 \%$ & \multicolumn{2}{|c|}{$10 \%<\mathrm{Td}<20 \%$} & $\mathrm{~T}<10 \%$ \\
\hline Agou & 10 & 7 & 70 & 2 & 20 & 1 & 10 \\
Ave & 15 & 7 & 47 & 4 & 27 & 4 & 27 \\
Haho & 20 & 13 & 65 & 2 & 10 & 5 & 25 \\
Région & 45 & 27 & 60 & 8 & 18 & 10 & 22 \\
\hline
\end{tabular}

$\mathrm{N}=$ Nombre de troupeaux ayant connu des mortalités DV

\section{Les causes principales de la diarrhée Examen bactériologique}

L'examen macroscopique de vingt (20) échantillons de selle a révélé la présence de fèces pâteuses, sanguinolentes de couleur jaunâtre, parfois blanchâtre ou verdâtre.

L'examen microscopique a révélé la présence d'une flore intestinale assez abondante avec des bactéries à gram-négatif et gram-positif.

La coproculture sur milieux nutritifs a permis d'isoler six (06) germes de E. Coli et trois (03) germes de Salmonella sp.. 
Les tests sérologiques d'agglutination de 20 échantillons ont détecté neuf (09) anticorps de Salmonella $s p$ avec un titre d'anticorps T variant de $1: 100$ à $1: 800$. Aucun germe d'E. Coli n'a été isolé avec cette méthode (Tableau 7).

Tableau 7. Analyses bactériologiques

\begin{tabular}{|l|l|l|l|l|}
\hline \multirow{3}{*}{ Numéro veaux } & \multicolumn{2}{|c|}{ Réaction d'agglutination } & \multicolumn{2}{c|}{ Coproculture } \\
\cline { 2 - 5 } & $\begin{array}{l}\text { Salmonela } \\
\text { sp.(Titre } \\
\text { d'anticorps) }\end{array}$ & E. Coli & $\begin{array}{l}\text { Salmonela } \\
s p .\end{array}$ & E. Coli \\
\hline A2443 & - & - & - & + \\
\hline Veau sn & - & - & - & + \\
\hline Sw Nd & $1: 400$ & - & - & + \\
\hline Veau Bg & $1: 400$ & - & - & + \\
\hline Az1 & $1: 400$ & - & - & + \\
\hline A2452 & - & - & - & + \\
\hline A2448 & $1: 800$ & - & - & - \\
\hline TLBg & $1: 200$ & - & - & - \\
\hline A2431 & $1: 200$ & - & - & - \\
\hline A1134 & $1: 200$ & - & + & - \\
\hline TP Nd & $1: 100$ & - & + & - \\
\hline Ax & $1: 100$ & - & + & - \\
\hline A1890 & - & - & - & - \\
\hline A2365 & - & - & - & - \\
\hline A2460 & - & - & - & - \\
\hline Etsi & - & - & - & - \\
\hline A2436 & - & - & - & - \\
\hline Ets3 & - & - & - & - \\
\hline A32 & - & - & - & - \\
\hline Ets2 & - & - & - & - \\
\hline
\end{tabular}

$+=$ contrôle cas positifs $;-=$ contrôle cas négatifs

Les tests bactériologiques révèlent que : $60 \%$ des veaux $(\mathrm{n}=12)$ ont été contrôlés positifs aux germes de Salmonella sp. et d'Escherichia coli ; $40 \%$ $(\mathrm{n}=8)$ ont été contrôlés négatifs. Parmi les cas positifs, $60 \%$ ont hébergé les germes de Salmonella sp. et 30\% ont hébergé des germes d'Escherichia coli. Les deux germes (Salmonella sp. et Escherichia Coli) ont été hébergés par $30 \%$ des veaux.

\section{Examen coprologique}

Quatre (4) principaux parasites ont été identifiés: il s'agit des Coccidies sp., des Strongles spp. ,des Strongilö̈des et des Trichirus avec des OPG moyens respectifs de 120,200,567,100.

- $50 \%$ des veaux ont été infestés de Coccidies sp. avec un OPG moyen de 120 .

- $50 \%$ des veaux ont été infestés de Strongles sp avec un OPG moyen de 200 . 
- $15 \%$ des veaux ont été infestés de Strongiloïdes avec un OPG moyen de 567.

- $15 \%$ des veaux ont été infestés de Trichuris avec un OPG moyen de 100 .

- 1 veau a hébergé les 4 parasites (Coccidies sp. Strongle sp. Strongylö̈des sp.Trichuris).

- 9 veaux ont hébergé 2 parasites (Coccidies sp. Strongle sp.).

- 4 veaux ont hébergé 1 seul parasite (Strongles sp.).

- Les Strongles et les Coccidies ont été les principaux parasites gastrointestinaux qui ont infesté les veaux.

\section{Discussion}

Au regard des différents modes d'élevage et au vu des résultats de laboratoire il apparait clairement que l'origine de la DV est à la fois endogène (microbes) et exogène (facteurs favorisants) (Bradford \& Smith, 2008 ; Dalton et al., 1972 ; Fisher \& Delafunte, 1972 ; Ravary \& Sattler, 2006). Certes, la diarrhée des veaux a des origines multiples (Hunter et al., 1994; Amtutz 1965; Planchenault, 1981; Mae, 2010). Certains auteurs ont estimé qu'elle n'est pas une pathologie, mais le symptôme d'une maladie (Roy et al., 2006). Les résultats bactériologiques et parasitologiques de nos travaux ont révélé que la principale cause de la diarrhée des veaux a été les infections par les entérobactéries d'E. Coli et de Salmonelle sp. et les enquêtes épidémiologiques ont révélé que les causes favorisantes ont été prioritairement la mauvaise conduite de l'élevage, l'hygiène, l'insuffisance de suivi zoosanitaire et la pluri-paternité des troupeaux. Ces résultats ont été soutenus par les auteurs, Lorino et al. (2005) et Ewy et al. (2005) qui ont estimé que la diarrhée des nouveaux nés est souvent due aux manques d'hygiène et aux mauvaises conduites du troupeau.

Les travaux ont révélé que les facteurs favorisants ont sensiblement contribué aux taux de mortalité des veaux ; ceux-ci ont été remarqués à travers le mode d'élevage dans les catégories de troupeaux A, B, C (Tableau 3). Dans ces troupeaux, les troupeaux de catégorie A ont enregistré le taux de mortalité le plus élevé $(\mathrm{Td}>20 \%)$; ce taux a dépassé celui de la catégorie $\mathrm{C}(\mathrm{Td}<10 \%)$ où les conditions hygiéniques ont été meilleures. En effet dans les troupeaux de catégorie A l'eau des barrages, des marres et des rigoles constituent les principales sources d'eau de boisson. L'habitat est constitué d'abri de fortune fait de branches d'arbre. Les veaux suivent leur mère au pâturage et sont soumis aux diverses pressions pathologiques et de fatigue physique. Les soins vétérinaires sont exécutés par les éleveurs eux-mêmes et se limitent en des administrations de capsules de tétracyclines et de certaines herbes et écorces de bois en cas de maladies. Ces troupeaux sont essentiellement des troupeaux regroupés provenant de divers horizons dont le bouvier n'en est pas toujours 
propriétaire (Adanlehoussi \& Kotoé, 2005). Cette catégorie qui représente $60 \%$ des troupeaux avec un taux de mortalité supérieure à 20\% constitue la catégorie d'élevage extensive traditionnelle (Ravary \& Sattler, 2006; Mae, 2005).

Quant aux troupeaux de catégorie $\mathrm{C}$, les veaux restent en enclos construits en bois et couverts de tôle ou de paille. Le sol est parfois damé ou cimenté. L'eau de boisson est constituée d'eau de pluie, de puits ou de cours d'eau. Les veaux restent sous corde ou dans un parc dans la journée tandis que les mères sont conduites au pâturage. Au retour le soir, les veaux sont libérés et profitent du lait de leur mère. Cette pratique dure environ deux mois puis les veaux suivent les adultes au pâturage. Les éleveurs distribuent parfois quelques poignées de compléments alimentaires à leurs animaux le soir au retour du pâturage. La propriété des enclos pour les veaux est assurée hebdomadairement; les soins sanitaires sont rares mais réalisés par des techniciens. Dans la plupart de ces élevages le bouvier est propriétaire du troupeau et la traite du lait est régulière. Cette catégorie de troupeau représente $14 \%$ des élevages et le taux de mortalité dû à la DV est de $10 \%$. Il constitue la catégorie d'élevage laitier plus ou moins moderne (Adanlehoussi \& Kotoé, 2005 ; Bradford \& Smith, 2008 ; Mohler et al., 2009).

Les troupeaux de catégorie $\mathrm{B}$, par contre, représentent la catégorie intermédiaire entre A et $\mathrm{C}$. Ici, les bouviers sont des agropasteurs et ne consacrent que 50\% de leur temps à leur bétail. La conduite des troupeaux est faite par des enfants non scolarisés (Adanlehoussi \& Kotoé, 2005). La traite du lait n'est pas obligatoire et la majorité des éleveurs sont propriétaires des troupeaux (Tableau 3).

Les études ont par ailleurs révélé que le taux de mortalité dû à la DV est lié aux classes d'âge des veaux et ceux âgés de 0 à 3 mois ont été les plus vulnérables (Ravary \& Sattler, 2006; Bradford \& Smith 2008). Par ailleurs, cette mortalité a diminué progressivement à mesure que l'âge avance $(53 \%$ entre 0 et 3 mois contre 4\% en 6 mois et plus). Les travaux de Planchenault (1981) dans le ranch du Mali ont connu un taux supérieur de 18\% sur des veaux âgés de 0 à 1 an. Selon les auteurs, Amstutz (1965), et Mae (2010) les infections microbiennes ne sont pas les seuls responsables de la diarrhée, mais aussi la prise du colostrum par le nouveau-né est nécessaire dans les premiers jours de naissance du veau pour augmenter son immunité. Une négligence peut être l'une des causes favorisantes de la diarrhée car le colostrum permet au veau de résister aux maladies durant les premières heures de naissance. Les mêmes observations ont été faites par Planchenault (1981) au ranch de Madima Diassa au Mali chez les veaux âgés de 0 à 12 mois. Les auteurs Boka et al. (2019), Bengaly et al. (1998), et Desquesnes et al. (1999) ont ajouté que la résistance aux maladies dépend de l'immunité acquise de la mère et que l'immunité décline en fonction de l'âge. Desquesnes et al. (1999), expliquent 
cette diminution par l'existence d'une immunité maternelle acquise qui protège les jeunes sujets des infestations tandis que chez les plus âgées, elle est sujette à un déclin et fait place à une immunité acquise suite au contact avec les microbes. Bradford \& Smith (2008) ont soutenu que la durée de la protection passive liée au colostrum est relativement courte, mais en considérant que de nombreux veaux sont exposés aux salmonelles dans leurs premières semaines de vie, la protection colostrale peut être utile. Or, dans toutes les catégories de troupeaux de cette étude et plus particulièrement à la station Avetonou, la prise du lait par le veau dans les premiers moments de naissance n'a pas été rigoureuse; c'est ce qui explique le taux élevé de prévalence de la DV observée à Avétonou. Quant à la préfecture d'Agou la forte prévalence proviendrait $d u$ fait que la station a longtemps abrité un important effectif de bovins et avait servi de centre de vulgarisation d'élevage bovin dans la préfecture et ses environs dans les années 1980. Il est probable qu'elle soit l'épicentre de la propagation de cette maladie (Figure 1).

Les résultats des travaux de laboratoire ont révélé que les selles ont un aspect pâteux, sanguinolent, de coloration jaunâtre, ou blanchâtre (Mae, 2010; Caillot, 2006 ; Jigou, 2006). Les auteurs, Planchenault (1981) et Ravary et Sattler (2006) ont affirmé que la diarrhée des veaux de couleur blanc jaunâtre est provoquée par E. coli. Pour Fichou (2003), Gelbery (2001), Quin (2002), Ravary et Sattler (2006), la diarrhée de couleur jaunâtre est le plus souvent causée par des salmonelles et est fatale dans cent pour cent des veaux atteints. Les travaux de ces auteurs sont conformes aux résultats de cette étude selon lesquels les entérobactéries (Salmonela sp. et E. Coli $s p$ ) pourraient être considérées comme les principaux responsables de la diarrhée des veaux. Fichou (2003), Hunter et al. (1994) et Ravary et Sattler (2006) ont estimé que la majorité des mortalités néonatales sont causées par des colibacilles et des salmonelles. Certes, ces microbes font partie des entérobactéries normales du tube digestif des ruminants d'après Smith et Linggood (1972) et Contrepoids et Gouet (1983), mais ils deviennent pathogènes soit à cause de la souche, ou de leur localisation dans le tractus digestif (Phillips, 1971) ou soit ils peuvent s'associer à d'autres bactéries pour mener des activités synergiques en produisant des toxines (Amstutz, 1965 ; Morin et al., 1976).

Les résultats bactériologiques sont diversement appréciés grâce â 1' interprétation selon la méthode d'analyse et la charge bactérienne de l'infection. La méthode d'agglutination n'a pas été efficace pour la détection d'E. Coli. Par contre, la coproculture a permis de détecter six (06) cas, soit $30 \%$ d'infestation par E. Coli. Pour le test d'agglutination, deux veaux marqués TP Nd et Ax ont présenté des titres d'anticorps de Salmonella $\mathrm{T}<1$ : 100 ; cette charge est considérée faible car elle est inférieure à la charge minimale létale pour un veau (Bradford \& Smith, 2008) quand bien même les veaux ont présenté les symptômes de diarrhée. Les résultats de la présente 
étude sont conformes à ceux de Parreno et al. (2004) qui ont confirmé que ces faibles anticorps seraient ceux que les veaux avaient acquis auprès de leur mère depuis la période fœtale et qui ont tendance à disparaître dès leurs premiers jours de naissance. Trois (03) veaux marqués $\mathrm{Sw} \mathrm{Nd}$, Veau Bg et Azl ont présenté un titre d'anticorps $T \geq 1: 400$ et un $(01)$ veau marqué $A$ 2448 a présenté un titre $\mathrm{T} ; \mathrm{T} \geq 1: 800$. Ces veaux par ailleurs ont été infestés à la fois par E. Coli et Salmonella; ces germes sont susceptibles de provoquer la maladie car ils sont plus proches de la charge bactérienne, pouvant causer la mort des veaux qui est $\mathrm{T} \geq 1: 200$. Les veaux marqués $\mathrm{A} 1134, \mathrm{TPNd}$ et Ax, aux titres d'anticorps $\mathrm{T}<1: 200$ et contrôlés positifs à la fois par le test d'agglutination et de coproculture pour les germes de Salmonella sp. et E. Coli sont considérés positifs. Cependant le taux d'anticorps est jugé faible, par conséquence, ces germes seraient à l'origine du symptôme de la DV mais ils ne sont pas à l'origine de la mort des veaux suite à la DV. Bradford \& Smith (2008) rapporté par Maes ont estimé que la dose létale pour la souche virulente de salmonelle est de $10^{7}$ bactéries par gramme de fèces pour un veau âgé de 6 à 7 semaines et de $10^{10}$ pour un veau de 12 à 14 semaines d'âge. Les huit (8) derniers veaux marqués : A1890, A2365, A2460, Etsi, A2436, Ets3, A32 et Ets2, qui avaient cliniquement manifesté la maladie mais ont été contrôlés négatifs à la fois aux deux tests (Salmonella et d'E. Coli) nous laissent croire que la DV pourraient avoir d'autres origines comme l'ont affirmé Planchenault (1981), Bradford et Smith (2008) qui affirment que très souvent les virus (Corona virus, Rota virus, Adénovirus et Bovine Virous Diarrhea (BVD) sont souvent à l'origine de la diarrhée néonatale.

Quant aux travaux d'analyse coprologique, les résultats ont révélé une infestation parasitaire de strongles et de coccidies mais avec une charge parasitaire moyenne assez faible $(100<\mathrm{OPG}<600)$ par rapport à la charge normale tolérée par un veau $(400<\mathrm{OPG}<1000)$ (Bradford \& Smith, 2008 ; Fichou, 2003 ; Balley, 1974) par conséquent les parasites identifiés dans les prélèvements des échantillons des veaux ne sont pas considérés ni comme causes principales ni comme un facteur favorisant de la diarrhée.

\section{Conclusion}

La diarrhée des veaux constitue l'une des contraintes majeures dans les élevages bovins car elle empêche une bonne évolution du troupeau. L'évaluation de la situation sanitaire des veaux et la détermination des causes de la diarrhée sont d'une grande importance pour la relance de l'élevage bovin. $\mathrm{Eu}$ égard aux résultats bactériologiques, coprologiques et d'enquête, il est possible d'estimer que les Salmonella sp et E. Colis sp. ont été les principaux auteurs de la diarrhée des veaux avec un taux de mortalité assez élevé et dont les causes favorisantes seraient le manque d'hygiène et le mode d'élevage pratiquée dans les troupeaux. Certes, ces microorganismes font partie des 
microflores normales du milieu gastrique des ruminants. Cependant, il est nécessaire d'identifier le sérotype de ces entérobactéries. D'autre part, le caractère étiologique multifactoriel de la maladie permet de soupçonner la coinfestation des bactéries et des virus, en particulier le rota virus, le corona virus et le (BVD). Des études virologiques doivent être menées pour parfaire ces travaux.

\section{Remerciements}

Nous remercions le personnel des laboratoires de la station d'Avétonou, du service vétérinaire d'Accra et du centre hospitalier préfectoral de Kpalimé pour leur collaboration et leur technicité pour la réalisation de cette étude. Nos remerciements vont également aux bouviers de la section zootechnie et aux chercheurs de la station Avétonou pour leur apport physique, moral et intellectuel.

\section{References:}

1. Adanlehoussi, A. \& Kotoé, M. (2005). Elevage bovin. In Productions animales au Togo: Situation de référence. Institut Togolais de Recherche Agronomique (ITRA) Togo, 127p.

2. Amstutz, H.E. (1965). Occurrence and etiology of infectious calf diarrhea. J.A.V.M.A. pp.147, 1360

3. Anderson, R. J., House, J. K., \& Smith, B. R. (2001). Epidemiology and biological characteristics of salmonellosis in three dairy herds. J. Am. Vet. Med.Assoc. 19, 310-322

4. Balley, W.S. (1974). Le parasitisme chez le veau; In Chirurgie et Médecine des bovins, 19, 310-322

5. Bengaly, Z. G. R., Sidibe, I., \& Duvallet, G. (1998). Infections trypanosomiennes chez des bovins dans la zone Sud-soudanienne du Burkina Faso. Revue Elev. Med. Vet. Pays trop, 51(3): 225-229.

6. Boka, O.M., Boka, E.E.J., Yapi, G.Y., Traoré, S.I., Kouamé, K.E. (2019). Epidémiologie de la trypanosomose animale africaine chez les bovins dans le département du Korhogo (Côte d'Ivoire). Revue d'élevage et de médecine vétérinaire des pays tropicaux, 72 (2): 83-89

7. Braford \& Smith (2008). Large animal internal medicine. 4th edition. Mosby. 1872p.

8. Caillot, D. (2006). Institut de l'élevage. Etude du département. Action régionale. $11 \mathrm{p}$

9. Contrepoids, M. \& Gouet, PH. (1983). Etiologie des colibacilloses chez les bovins. Rec. Méd. Vét., 159(3) : 159-166. 
10. Dalton, R. G, Ficher, E. W., \& Intyre, W. J. M. (1972). Charges in blood chemistry, body weight and hematocrit of calves affected with neonatal diarrhea. Bull. Vet. Jn., 121, 34-31

11. Desquesnes, M. M. J. F., de La Rocque, S., Solano, P., Millogo, L., Bengaly, Z., \& Sidibe, I. (1999). Enquête parasitologique et sérologique (Elisa-indirect) sur les trypanosomoses des bovins dans la zone de Sidéradougou, Burkina Faso. Revue Élev. Méd. vét. Pays trop, 54(3-4) : 223-232.

12. Dufrasne, V. (2003). Diarrhée néonatale des veaux et réhydratation par voie orale. Thèse de doctorat en Med. Vet. Faculté de médicine de Créteil ; 181p.

13. Ewy, A., Neff, K., \& Sutter-Luth B. (2005). Diarrhée des veaux : mesures de lutte. Revue UFA, 9/5

14. Fichou, E. (2003). Enquête de terrain sur l'étiologie microbienne des diarrhées néonatales des veaux et sur la sensibilité à l'anti-infectieux des colibacilles isolés. Thèse de doctorat de médecine vétérinaire, Nantes, $104 p$

15. Fisher, E.W. \& Delafunte, H. (1972). Water and electrolyte study in the newborn calves with particular reference to the effect of diarrhea. Res. Vet. Sci., 13, pp 315-332.

16. Gelbery, H.B. (2001). Alimentary system. In: Thomson's special veterinary pathology 3rd edition.St. Louis (Mo) : Mosby, Inc., pp 179.

17. Hunter, A., Uilenberg, G., \& Meyer C. (1994). La santé animale. In : agriculture tropicale en poche ; $288 \mathrm{p}$.

18. Jigou, V. (2006). Diarrhées des veaux. In santé des veaux, 9, 1-10.

19. Lorino, T., Daudin, J. J., Robin, S., \& Sana, M. (2005). Factors associated with time to neonatal diarrhea in French beef calves. Prev. Vet. Met. 68, 91-102.

20. Mae, P. (2010). Etiologie des diarrhées néo-natale et transfert colostrale chez le veau : enquête dans la creuse. Thèse de doctorat vétérinaire ; Ecole nationale d'Alford $124 \mathrm{p}$

21. Mohler, V., Izzo, M.M., \& House, J.K. (2009). Salmonella in calves. Vet. Clin, Foot Anim.; 25, 37-54.

22. Morin, M., Lariviére, S., \& Lallie, R. (1976). Pathological and microbiological observation made on spontaneous cases of acute neonatal calf diarrhea. Can.J. comp. Med., 40, 228-240.

23. Parreno, V., Béjar, C., Vagnozzi, A., Barrendeguy, M., \& Constantini, V. (2004). Modulation by colostrum-acquired maternal antibody of systemic and mucosal antibody responses to rotavirus in calves experimentally challenged with bovine rotavirus. Vet. Immunopathology, 2004, 100, 7-2. 
24. Phillips, R.W., Lewis, L.D., \& Knox, K. L. (1971). Alteration in body water turn-over and distribution in neonatal calves with acute diarrhea. Ann. N. Y. acad. Sci., pp. 176, 231

25. Planchenault, D., Bréaud, A., Sartirana, E., \& Tacher, G. (1981). Entérite infectieuse du veau à E.Coli dans le ranch du Mali in Rev. d'Elevage et de Méd. Vét. des paysTtropicaux. Vol. 34, (2)

26. Quin, P.J. (2002). Enterobacteriaceae. In Veterinary microbiology and microbiology disease. Carlton (Victoria, Australia): Blackwell science Ltd. Pp 106-123.

27. Ravary, B. \& Sattler, N. (2006). Néonatal du veau. $1^{\text {ere }}$ édition. Les éditions du point vétérinaire ; $265 \mathrm{p}$

28. Roy, C. \& Guerin, D. (2006). Les diarrhées des veaux sous la mère, $8 \mathrm{p}$.

29. Smith, H.W. \& Linggood, M.A. (1972). Further observations on E. coli enterotoxins with particular regard to those produced by atypical piglet strains and by calf and lamb strains: The transmissible nature of these enterotoxins and of K antigens passed by calf and lamb strains. J. Med. Microbiol. 1972, 5,243-250.

30. Thiempont, D., Rochette, F., \& Vanparsjs, O. F. (1995). Diagnostic de verminose par examen coprologique. Jansen research foundation. Beese, Belgique 203p.

31. Widal \& Félix (2009). Guide pratique des analyses médicales de Pascal Dieusaert. 5 ème Editions Maloine.

32. Willis, B. (1983). Helminthes et helminthoses des ruminants domestiques d'Afrique tropicale $378 \mathrm{p}$. 